\title{
Aberration correction in holographic optical tweezers
}

\author{
Kurt D. Wulff, Daniel G. Cole, Robert L. Clark \\ Center for Biologically Inspired Materials and Material Systems \\ Duke University, Durham, NC, USA \\ Roberto DiLeonardo \\ INFM-CRS SOFT clo Universita di Roma "La Sapienz", I-00185, Roma, Italy \\ Jonathan Leach, Jon Cooper, Graham Gibson and Miles J. Padgett \\ Department of Physics and Astronomy, University of Glasgow, Glasgow G12 8QQ, Scotland, \\ $U K$ \\ m.padgett@physics.gla.ac.uk
}

\begin{abstract}
Holographic or diffractive optical components are widely implemented using spatial light modulators within optical tweezers to form multiple, and/or modified traps. We show that by further modifying the hologram design to account for residual aberrations, the fidelity of the focused beams can be significantly improved, quantified by a spot sharpness metric. However, the impact this improvement has on the quality of the optical trap depends upon the particle size. For particle diameters on the order of $1 \mu \mathrm{m}$, aberration correction can improve the trap performance metric, which is the ratio of the mean square displacement of a corrected trap to an uncorrected trap, in excess of $25 \%$, but for larger particles the trap performance is not unduly affected by the aberrations typically encountered in commercial spatial light modulators.
\end{abstract}

(C) 2006 Optical Society of America

OCIS codes: (090.1000) Aberration compensation; (140.7010) Trapping

\section{References and links}

1. A. Ashkin, J. M. Dziedzic, J. E. Bjorkholm, and S. Chu, "Observation of a Single-Beam Gradient Force Optical Trap for Dielectric Particles," Opt. Lett. 11, 288-290 (1986).

2. Cell Robotics International Inc. (Albuquerque, USA); P.A.L.M. GmBH (Bernried, Germany); Arrxy Inc. (Chicago, USA).

3. J. E. Molloy and M. J. Padgett, "Lights, action: optical tweezers," Contemporary Physics 43, 241-258 (2002).

4. S. M. Block, D. F. Blair, and H. C. Berg, "Compliance Of Bacterial Flagella Measured With Optical Tweezers," Nature 338, 514-518 (1989).

5. J. T. Finer, R. M. Simmons, and J. A. Spudich, "Single Myosin Molecule Mechanics - Piconewton Forces and Nanometer Steps," Nature 368, 113-119 (1994).

6. M. D. Wang, H. Yin, R. Landick, J. Gelles, and S. M. Block, "Stretching DNA with optical tweezers," Biophys. J. 72, 1335-1346 (1997).

7. D. G. Grier, "A revolution in optical manipulation," Nature 424, 810-816 (2003).

8. J. Liesener, M. Reicherter, T. Haist, and H. J. Tiziani, "Multi-functional optical tweezers using computergenerated holograms," Opt. Commun. 185, 77-82 (2000).

9. J. E. Curtis, B. A. Koss, and D. G. Grier, "Dynamic holographic optical tweezers," Opt. Commun. 207, 169-175 (2002).

10. K. Visscher, G. J. Brakenhoff, and J. J. Krol, "Micromanipulation by Multiple Optical Traps Created by a Single Fast Scanning Trap Integrated with the Bilateral Confocal Scanning Laser Microscope," Cytometry 14, 105-114 (1993). 
11. J. E. Molloy, J. E. Burns, J. Kendrickjones, R. T. Tregear, and D. C. S. White, "Movement And Force Produced By A Single Myosin Head," Nature 378, 209-212 (1995).

12. P. Jordan, J. Leach, M. Padgett, P. Blackburn, N. Isaacs, M. Goksor, D. Hanstorp, A. Wright, J. Girkin, and J. Cooper, "Creating permanent 3D arrangements of isolated cells using holographic optical tweezers," Lab On A Chip 5, 1224-1228 (2005).

13. G. Sinclair, P. Jordan, J. Courtial, M. Padgett, J. Cooper, and Z. J. Laczik, "Assembly of 3-dimensional structures using programmable holographic optical tweezers," Opt. Express 12, 5475-5480 (2004).

14. G. Sinclair, P. Jordan, J. Leach, M. J. Padgett, and J. Cooper, "Defining the trapping limits of holographical optical tweezers," J. Mod. Opt. 51, 409-414 (2004).

15. T. Ota, S. Kawata, T. Sugiura, M. J. Booth, M. A. A. Neil, R. Juskaitis, and T. Wilson, "Dynamic axial-position control of a laser-trapped particle by wave-front modification," Opt. Lett. 28, 465-467 (2003).

16. E. Theofanidou, L. Wilson, W. J. Hossack, and J. Arlt, "Spherical aberration correction for optical tweezers," Opt. Commun. 236, 145-150 (2004).

17. K. Ladavac and D. G. Grier, "Microoptomechanical pumps assembled and driven by holographic optical vortex arrays," Opt. Express 12, 1144-1149 (2004).

18. www.physics.gla.ac.uk/Optics/projects, Submitted to Appl. Opt. 2005.

19. K. Visscher, S. P. Gross, and S. M. Block, "Construction of multiple-beam optical traps with nanometer- resolution position sensing," IEEE J. Quantum Electron. 2, 1066-1076 (1996).

20. M. Polin, K. Ladavac, S. H. Lee, Y. Roichman, and D. G. Grier, "Optimized holographic optical traps," Opt. Express 13, 5831-5845 (2005)

21. M. Born and E. Wolf, Principles of optics, 7th edition (Cambridge University Press, Cambridge, 1999).

22. J. R. Fienup and J. J. Miller, "Aberration correction by maximizing generalized sharpness metrics," J. Opt. Soc. Am. A 20, 609-620 (2003).

23. R. A. Muller and A. Buffington, "Real-time correction of atmospherically degraded telescope images through image sharpening,” J. Opt. Soc. Am. 64, 1200-1210 (1974).

\section{Introduction}

In 1986 Ashkin et al. demonstrated an optical technique for trapping micrometer-sized dielectric particles suspended in a fluid medium, now referred to as optical tweezing [1]. Optical tweezers can now be bought commercially [2] and have found numerous applications [3], particularly in the biological sciences. For example, tweezers have been used in measuring the compliance of bacterial tails [4], the forces exerted by motor proteins [5], and stretching DNA molecules [6].

Within the last five years optical tweezers have been revolutionized by the commercial availability of programmable spatial light modulators (SLM) [7]. In holographic optical tweezers, an SLM is configured to act as a phase-only diffractive optical element allowing a single incident laser beam to be split into many beams, each forming an individual optical trap. By updating the SLM with a sequence of holograms the individual optical traps can be moved independent of each other [8]. Considerable work has been reported on forming arrays of particles and, through appropriate hologram design, modifying the profile of individual traps [9]. Unlike earlier attempts to create multiple tweezers that used scanning mirrors or acousto-optic modulators to laterally scan the laser between traps [10,11], an SLM can also display holograms that act as additional lenses allowing for axial displacements. Both lateral and axial displacement of the multiple traps can be set independent of each other to create complicated 3D structures of micrometer-sized inert spheres or living cells [12] using a variety of hologram design algorithms [13].

The trapping mechanism in an optical tweezers arises from the intensity gradient near the beam focus. For this reason, beam quality is important for efficient optical trapping. The trapping force is maximized by using a high numerical aperture microscope objective lens, ideally designed to minimize unwanted aberrations and produce a diffraction limited point spread function (PSF). Any aberrations introduced elsewhere in the optical system, e.g., by the SLM or other optics, will increase the size of the PSF and could degrade trapping performance.

There are two types of SLMs: electrically addressed and optically addressed. Electrically addressed SLMs, the type used in these experiments, are based on a semiconductor array over-

$\# 68285$ - \$15.00 USD

(C) 2006 OSA
Received 27 February 2006; revised 14 April 2006; accepted 16 April 2006 1 May 2006 / Vol. 14, No. 9 / OPTICS EXPRESS 4171 
coated with a liquid crystal layer that produces a programmable phase retardation. The optical flatness of such devices is rarely better than a few optical wavelengths across their $10-20$ $\mathrm{mm}$ aperture. Optically addressed SLMs can have similar wavefront distortions associated with them. This is in contrast to a commonly used criteria for diffraction limited optics, which requires the RMS wavefront error to be less than $\lambda / 14$ achieving a Strehl ratio greater than 0.8 . So, although SLMs offer many advantages regarding multiple and modified trapping, their limited optical flatness can degrade beam quality.

Previous research has shown that aberrations associated with defocus can partially compensate for the aberrations associated with deep trapping [14] and, similarly, that a deformable mirror can introduce sufficient wavefront curvature to shift the axial position of the trap by 2 $\mu \mathrm{m}$ [15]. More recently, a deformable mirror has been used to correct for aberrations in a trap by using the two-photon induced, fluorescence signal from a trapped sphere as a measure of the beam's peak intensity, which is assumed to dictate the trap performance [16]. Aberration correction, applied using an SLM, has been used to improve the fidelity of optical vortices, whose transverse momentum components cause the circulation of micrometer-sized particles as a form of micro-pump [17].

In this work we show that the trapping stiffness of an optical tweezers can be improved by optimizing a spot sharpness metric for the beam's focus as back-reflected from the cover-slip. In addition, we show that the impact this optimization has on the trap stiffness is a function of particle size.

\section{Experimental Setup}

Our optical tweezers are based upon a Zeiss Axiovert 200 inverted microscope with a 100x objective lens, NA 1.3 (Zeiss Plan-Neofluar, oil-immersed, infinity-corrected). To form the trap we use a commercial Yd:YAG laser, frequency-doubled to give a maximum power of $3 \mathrm{~W}$ at $515 \mathrm{~nm}$ (VersaDisc). After expansion and collimation, the beam from this laser is reflected off a computer-controlled SLM (HoloEye 2500) and coupled into the microscope, directed upwards (inverted optical tweezers geometry) through the objective, and enters the water-filled sample cell though a cover-slip, creating an optical trap within the water. The plane of the SLM is imaged to the rear aperture of the microscope objective. The SLM was controlled using our standard software package [18], which was modified to include aberration corrections in the displayed holograms. The SLM produced two neighboring traps, one with aberration correction and one without, so that same sized particles could be compared in identical environments.

Displacement of the particle from the trap center can be measured with an accuracy of a few nanometers using either a quadrant photodiode $[5,19]$ or direct video microscopy [20]. The photodiode approach gives a measurement bandwidth of several kilohertz, whereas the video technique is restricted to video frame rates (10's Hz) but is simpler to setup and can be used to measure the motion of many particle simultaneously. The two trapped particles were tracked on a CCD camera (Q Imaging Fast 1394) using a centroid tracking algorithm implemented in LabView. The trapped particles were monitored over an interval of several seconds to establish the mean-square displacement of the beads from their respective trapping centers. A measure of the effect of the aberration correction on trapping stiffness can be made by taking the ratio of these two mean-square displacements; this metric is insensitive to laser power. This trap performance metric can be directly related to the change in trapping stiffness using the equipartition theorem for a particle bound in a harmonic potential, $\left\langle r^{2}\right\rangle=k_{B} T / k$ ( $k$ is the trap stiffness, $k_{B}$ is Boltzmann's constant and $T$ is the absolute temperature of the surrounding fluid), so that the trap performance metric is described by Eq. 1 .

$$
M_{p}=\frac{\left\langle r^{2}\right\rangle_{\mathrm{c}}}{\left\langle r^{2}\right\rangle_{\mathrm{u}}}=\frac{k_{\mathrm{u}}}{k_{\mathrm{c}}}
$$

\#68285 - \$15.00 USD

(C) 2006 OSA
Received 27 February 2006; revised 14 April 2006; accepted 16 April 2006 1 May 2006 / Vol. 14, No. 9 / OPTICS EXPRESS 4172 
Aberrations in the optical system result in wavefront distortion, $W$, at the exit pupil, which is a change in the phase of the wave front $\Delta \phi=k W=2 \pi(W / \lambda)$ [21]. The wavefront distortion can be expanded as a linear combination of basis functions. A convenient orthogonal basis is the set of Zernike polynomials, $Z_{n}^{m}: n$ is the radial order and $m$ is the azimuthal frequency. Second order modes $(n=2, m=\{-2,0,2\})$ are quadratic and characterize common aberrations of focus and astigmatism, and are the primary interest in this investigation due to the overall curvature of the SLM surface. Higher order modes, e.g., coma, trefoil, and spherical, and other aberrations, were investigated as well, but any quantifiable impact was difficult to discern.

The Shack-Hartmann sensor is the state of the art in wavefront sensing, but is difficult to incorporate into the trap design. As an alternative we used a sensorless technique to quantify the effect changes in aberration correction had on the PSF and trapping focus. The cross-section of the focused beam as reflected from the cover-slip was imaged on the CCD camera. A spot sharpness metric is defined according to Eq. 2,

$$
M_{s}=\left(\sum_{i j} I_{i j}\right)^{2} / \sum_{i j} I_{i j}^{2}
$$

where $I_{i j}$ is the intensity of the $i j$ th pixel; lower values correspond to a more tightly focussed spot [22]. It can be shown that this metric is a global minimum when all aberrations have been corrected [23].

\section{Results}

In order to establish a correlation between the spot sharpness and trap performance metrics, that is, between the focal spot size and the trapping stiffness, both metrics were measured while changing each of the astigmatism modal coefficients while keeping the other coefficient zero. In this case, the full range of the astigmatism coefficient corresponds to a maximum amplitude of $\pm 10 \lambda$ across the aperture of the SLM. For a $0.8 \mu \mathrm{m}$ diameter sphere, Figs. 1(a) and (d)

\section{Spot Sharpness}

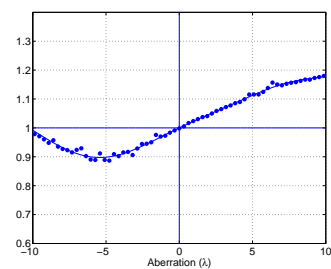

(a)

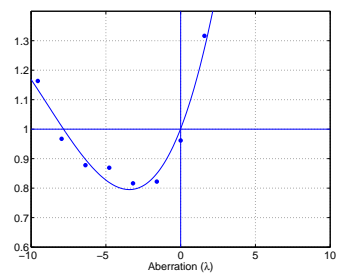

(d)

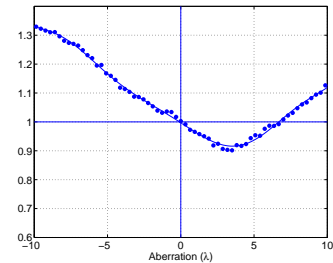

(b)

Trap Performance

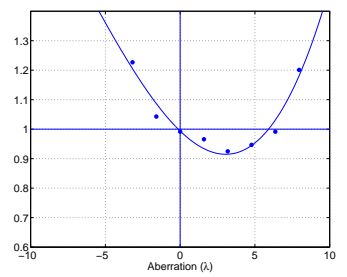

(e)

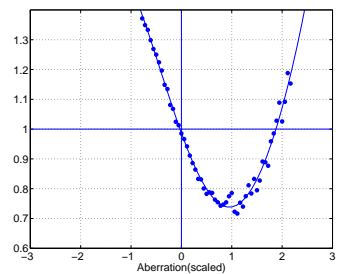

(c)

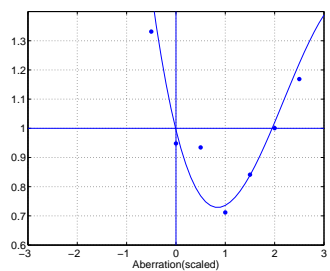

(f)

Fig. 1. Sharpness and performance metrics for a $0.8 \mu \mathrm{m}$ bead as a function of: (a,d) $Z_{2}^{-2}$ aberration, (b,e) $Z_{2}^{2}$ aberration, (c,f) and versus scaled changes in the linear combination of the two aberration terms.

$\# 68285$ - \$15.00 USD

(C) 2006 OSA
Received 27 February 2006; revised 14 April 2006; accepted 16 April 2006

1 May 2006 / Vol. 14, No. 9 / OPTICS EXPRESS 4173 


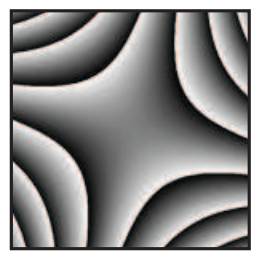

(a)

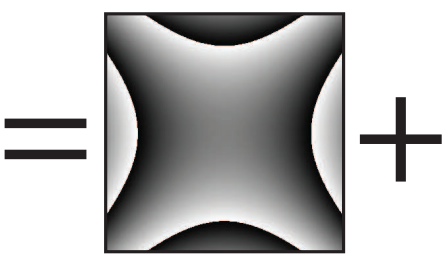

(b)

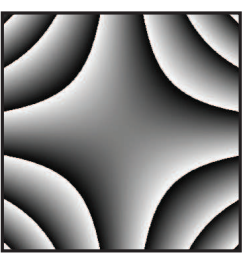

(c)

Fig. 2. Holograms used to correct relative aberrations. (a) The linear combination of (b) $Z_{2}^{2}$ aberration and (c) $Z_{2}^{-2}$ aberration. The linear combination hologram, (a), was the corrective hologram used in the later experiments. The black and white areas correspond to a phase of 0 and $2 \pi$ while the grey areas represent a phase of $\pi$.

show the spot sharpness metric and trap performance metric versus the $Z_{2}^{-2}$ astigmatism coefficient; Figs. 1(b) and (e) show the spot sharpness metric and trap performance metric versus the $Z_{2}^{2}$ astigmatism coefficient. For small changes in the aberration coefficients about the optimal values, the sharpness and trap performance metrics are quadratic, and the above measurements are enough to determine the shape of each quadratic surface. To further illustrate the quadratic dependence, each modal coefficient was fixed at the above minimizing value for that mode, and the linear combination was scaled. Figures 1(c) and (f) show the spot sharpness metric and trap performance metric versus scaled changes in this linear combination. Comparing these graphs shows a strong correlation between the spot sharpness metric and the trap performance metric. Figure 2 shows the two independent Zernike correction holograms and the linear combination of the two that was used for focal spot correction.

In order to investigate the impact of aberration correction on the trapping of particles of various sizes, the two astigmatism modes were combined into a single holographic correction applied to the SLM. Figure 3 shows the corrected and uncorrected focal spots of the trapping laser with $0.8,2$ and $5 \mu \mathrm{m}$ diameter circles superimposed for comparison. The net result is an symmetric spot for the corrected focal spot verse an asymmetric spot for the uncorrected focal spot.

We note that correction of the aberration terms leads to a $25 \%$ improvement in the trap performance metric of smaller particles, as can be seen in Fig. 1(f), an improvement directly related to an improvement in the trapping stiffness. However, for larger particles the trap performance is not improved as much by the correction. Comparing the focal spot size to the particle size leads us to conclude that if the PSF is smaller than the trapped particle then further optimization results in little improvement of the trap.

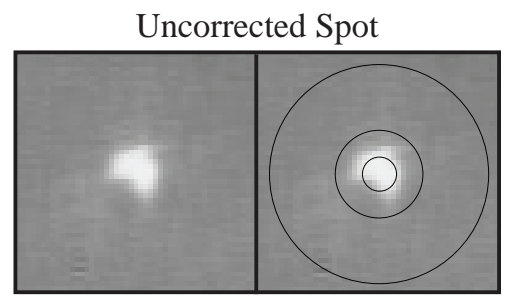

(a) (b)

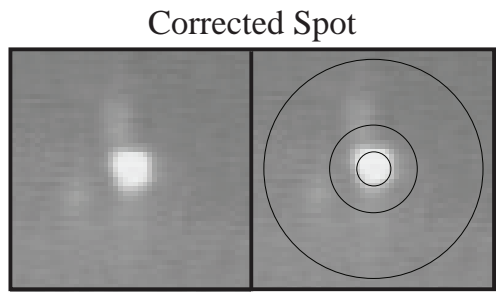

(c) (d)

Fig. 3. Laser focal spots for: (a) uncorrected spot (c) and corrected spot. (b,d) These show the same focussed laser spots with the three sized microspheres $0.8,2$ and $5 \mu \mathrm{m}$ spheres superimposed on them. The uncorrected laser spot falls outside the $0.8 \mu \mathrm{m}$ diameter bead. 
Uncorrected

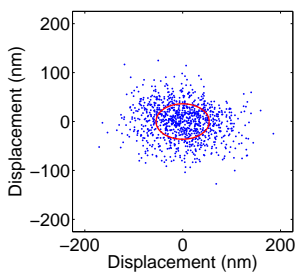

(a)

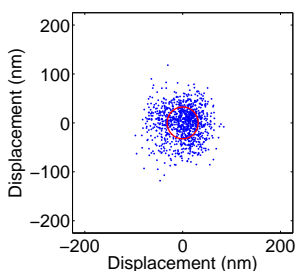

(d)

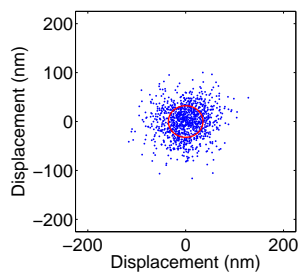

(b)

Corrected

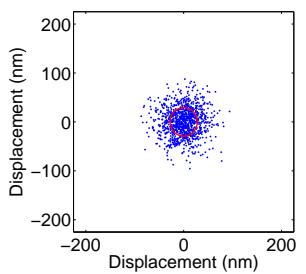

(e)

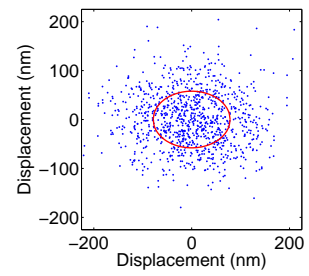

(c)

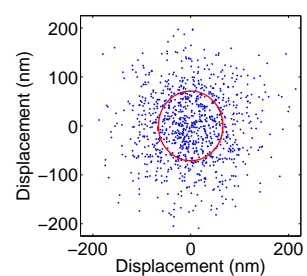

(f)

Fig. 4. Particle displacements for uncorrected and corrected traps for particle sizes of: $(a, b)$ $0.8 \mu \mathrm{m}$ (c,d) $2 \mu \mathrm{m}$, and (e,f) $5 \mu \mathrm{m}$. The superimposed ellipses show the root mean-square displacements. The corrected traps have circular RMS displacements giving a more uniform trap.

Figure 4 shows the recorded positions of the various sizes of particles, with and without aberration correction as measured over a period of $60 \mathrm{~s}$. The relative change in the measured RMS response for the corrected verse the uncorrected result in a 30\%,16\% and $0.5 \%$ improvement for the $0.8,2$ and $5 \mu \mathrm{m}$ silica microspheres respectively. In addition to showing explicitly that the improvement is greatest for the small particles, it also illustrates that residual astigmatism in the uncorrected trap leads to a corresponding elliptical symmetry in the motion of the particle.

\section{Conclusion}

In this paper we have shown that in addition to their use in the creation of multiple and modified optical traps that spatial light modulators can also be used to correct for aberrations within the optical train resulting in an improved trapping performance. Typically an electrically addressed SLM may deviate from flatness by up to $4 \lambda$, dominated by astigmatism due to the overall curvature of the SLM surface. This astigmatism may be corrected by adding the appropriate hologram to the SLM display resulting in a dramatic improvement in the fidelity of the focussed spot. The impact that this correction has on the performance of the optical trap is most noticeable for small particles. For the SLM used in this study, the improvement in trap performance for a $0.8 \mu \mathrm{m}$ diameter particles can be in excess of $25 \%$. However, for $5 \mu \mathrm{m}$ diameter particles our results show an improvement of less than $0.5 \%$. This dependence upon particle size is most likely associated with the relative size of the PSF and the trapped particle. Once the PSF is significantly smaller than the particle diameter, further reduction brings little improvement in trap performance. 